\title{
How phonetic is selective adaptation? Experiments on syllable position and vowel environment
}

\author{
ANTHONY E. ADES \\ Massachusetts Institute of Technology, Cambridge, Massachusetts 02139
}

\begin{abstract}
In several cases, the acoustic cues underlying a given phoneme depend heavily on its context. The selective adaptation method (Eimas \& Corbit, 1973) was used to investigate the existence of detectors sensitive to consonants in any environment, irrespective of their acoustic properties. The first experiment concerned consonants in initial and final position. Repeated presentation of a CV syllable had an adapting effect on a CV continuum but not on a VC continuum. The converse was also true. In a second experiment, a detector for $C_{1} V_{1}$ was fatigued by repeated presentation of $C_{1} V_{2}$, even though the adapting $\mathbf{C}_{1}$ had very little acoustically in common with the test $\mathbf{C}_{1}$. It is concluded that there are detectors for phonemes, or possibly features, which respond to a fairly abstract representation of the input, but not so abstract that a $\mathbf{C}$ is represented the same in initial and final position.
\end{abstract}

In several cases, the acoustic cues underlying a given phoneme are heavily dependent on the immediate context of that phoneme. This lack of invariance is largely the result of the way the speech articulators are programmed and of their inertial properties. What type of perceptual device can operate in the face of this fickle relationship between the waveform and its phonological representation?

The issue has been investigated in several ways. Experiments on synthetic speech have demonstrated that formant transitions are sufficient cues to the place of articulation of stop consonants in CV syllables (Cooper, Delattre, Liberman, Borst, Gerstman, 1952; Delattre, Liberman, \& Cooper, 1955). This means that the cues for place of articulation, which distinguishes labial, dental, and velar consonants, depend on the following vowel. By way of finding an acoustic basis for these facts, one might characterize the second and third formant (F2 and F3) transitions as rising in labials, falling in dentals, and diverging in velars. This covers much of the data, but cannot account for those dentals that can be synthesized without any falling transitions (Delattre et al, 1955), nor for the differences between initial and final consonants.

Tape splicing experiments, where the consonantal cues are spliced off from the following vowel and presented for identification alone (Fischer-Jorgensen, 1972) or spliced into other vowel contexts (Cole \& Scott, 1971), have tended to affirm the existence of invariant phonemic cues in natural speech. Direct studies of the spectral characteristics of natural speech (Halle, Hughes, \& Radley, 1956; Fischer-Jorgensen, 1954) have reached the same conclusion. However, they have generally found that formant transitions were the important cue in some cases, and the burst in others.

*This research was supported by Research and Training Grant in the Neural Sciences 72-4-1 to H.-L. Teuber from the Alfred $P$. Sloan Foundation, and by NIH Research Grant 05168-03. The author thanks M. F. Garrett, A. M. Liberman, K. N. Stevens, and E. C. T. Walker for their advice on the manuscript.
Stevens (1973) has given a more unified account of the acoustic basis for place of articulation distinctions in natural speech in terms of the rising, falling, and spreading frequency patterns associated with initial labials, dentals, and velars, respectively; he also suggests a treatment of the variability arising from acoustic differences between initial, medial, and final consonants, in terms of a learning model.

However, formulations of possible acoustic invariants in natural speech do not prove that the perceiver needs them to decode the speech stream, and the synthetic speech results in fact suggest that the perceptual representation of speech is at an abstract level which bears no straightforward $1: 1$ relation to acoustic attributes. One way to study the nature of the perceiver's representation is to contrast the effects of acoustic similarity with those of phonetic similarity: to do this, we must, of course, choose "minimal pairs" of stimuli that are similar acoustically but not phonetically, or vice versa.

A convenient experimental technique, selective adaptation, has been recently developed for use in speech research by Eimas and Corbit (1973). Eimas and Corbit presented the synthetic sound $/ \mathrm{da} /$ repeatedly to Ss, and then measured the position of their phoneme boundary on a continuum of synthetic $\mathrm{CV}$ s ranging from the voiced $/ \mathrm{da} /$ to the voiceless $/ \mathrm{ta} /$. The effect of this adaptation was to shift the phoneme boundary toward "D." Repeated presentation of $/ \mathrm{ba} /$, which, like /da/, contains a voiced stop, had the same effect./ta/ and $/ \mathrm{pa} /$, both voiceless, shifted the boundary in the opposite direction, toward the " $T$ " end of the series. The conclusion that there are a pair of "linguistic feature detectors," selectively sensitive to voiced and voiceless stimuli, respectively, appears to be justified. To this, we can add the findings of Cooper (1973) and Cooper and Bloomstein (1974), which point to a detector for a feature "labial"; testing on a bae/dae continuum, they have found that repeated presentation of $/ \mathrm{bi} /, / \mathrm{pae} /$, 

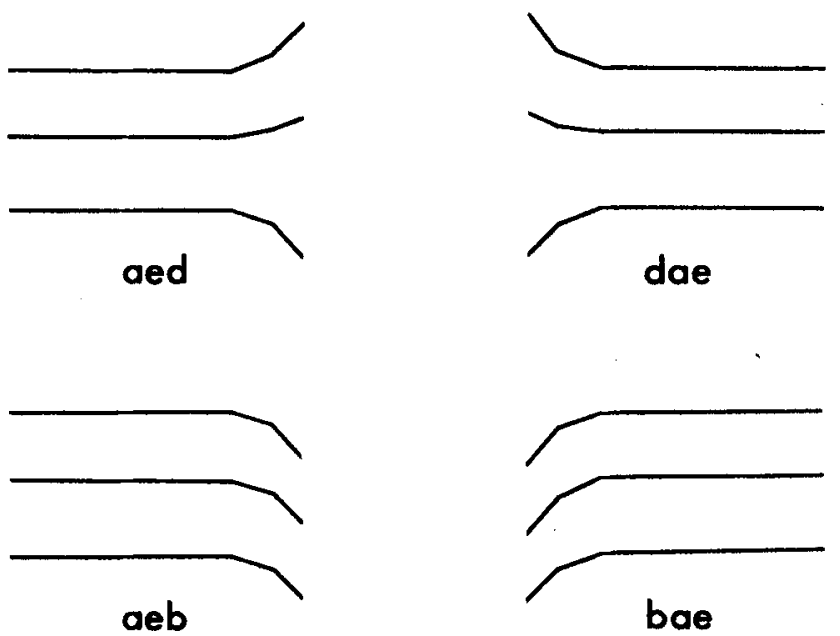

Fig. 1. Schematic spectrograms of the extreme stimuli on the aeb/aed and bae/dae test continua. These stimuli were also used as the adapting sounds in Experiment $I$.

/mae/, and /vae/, all labials, have a "B-adapting" effect.

However, as they stand, these experiments cannot tell us whether the fatigued detectors are specific for linguistic distinctive features or for certain subspeech attributes which may subserve them. The reason for this is that in both cases, voicing in initial stops and labials in initial position, the acoustic cues are relatively constant in different environments. Accounts of what these acoustic cues might be have been given by Lisker and Abramson (1970) and Stevens and Klatt (1971) for voicing and by Stevens (1973) for labials. Thus, the possibility of adaptation having taken place at a nonphonetic, auditory level cannot be ruled out. What is needed is a case where the fatiguing and test stimuli are phonetically the same, but acoustically different.

The two experiments reported here are intended to be more critical tests of the hypothesis that the detectors revealed in the adaptation technique are sensitive to the presence of linguistic distinctive features, as opposed to auditory attributes. In both experiments, the adapting sounds are phonetically similar to the sounds at one end of the test continuum, but at an auditory level may be considered closer to the sounds at the other end of the continuum. Place of articulation distinctions are used since it is in this area that acoustic and phonetic properties may be most easily teased apart. The first experiment concerns the adapting effect of $/ \mathrm{bae} /$ and /dae/ on an aeb/aed test series, and vice versa, and the second concerns the effect of certain examples of the syllable/de/ on a bae/dae series.

\section{EXPERIMENT I INITIAL AND FINAL POSITION}

Two continua of synthetic speech sounds were constructed, one ranging from $/ \mathrm{bae} /$ to $/ \mathrm{dae} /$, the other from /aeb/ to /aed/. Within each series, the sounds differed only in their F2 and F3 transitions. Because the two series are mirror images of each other in time, transitions which rise in the syllable /bae/, for example, must fall in the syllable /aeb/. The purpose of the experiment was to see what effect repeated presentation of say /bae/ would have on the perception of the aeb/aed series. If a phonetic, or feature, theory is correct, repetition of /bae/ should have a B-adapting effect on aeb/aed. If, instead, the detectors are only listening for rising vs falling patterns of frequency change, irrespective of context, then repeating /bae/ will weaken detectors for rising transitions and thereby effectively D-adapt an aeb/aed series.

\section{Method}

Stimuli. All the stimuli consisted of three formants. There were seven stimuli in each series, differing from each other only in the transitions of F2 and F3. In / bae/, both rise, and in /dae/, they both fall. In /aeb/, the steady state vowel is followed by falling transitions for $/ \mathrm{b} /$ and rising for $/ \mathrm{d} /$. Figure 1 shows schematic spectrograms of the extreme two stimuli on each continuum; these particular stimuli were used as adapting stimuli also. The transitions were $35 \mathrm{msec}$ in duration, and curved in two straight segments so that they completed two-thirds of their total trajectory in $15 \mathrm{msec}$ and one-third in $20 \mathrm{msec}$. Curved, rather than linear, transitions were used, since it proved difficult to synthesize final stop consonants without a burst with linear transitions. The relative distances between the adjacent stimuli on each continuum were in the ratio $2,1,1,1,1,2$, where one unit corresponds to $39 \mathrm{~Hz}$ in the extreme frequency of the $\mathrm{F} 2$ transition and $87 \mathrm{~Hz}$ in F3. The stimuli will be referred to as lying on a scale from 0 to 8 . The steady state frequencies of the formants were $742,1,618$, and $2,751 \mathrm{~Hz}$. Full details of the stimuli are given in the Appendix.

The test sounds were delivered from a Tandberg 3000X tape recorder, and the adapting sounds from a tape loop, mounted on an Ampex PR10. A decade at tenuator modulated the output of the Ampex. Output from both recorders was fed into an AR amplifier set to MONO; thus, all stimuli were delivered binaurally. An oscilloscope attached to the output of the amplifier permitted the $E$ to adjust the intensities of the stimuli to an accuracy of \pm about $0.3 \mathrm{~dB}$. Test and adapting stimuli were presented at the same intensity. Ss heard the stimuli over Telex headphones. They used the same headphones in the same orientation on every occasion.

Procedure. There were eight experimental sessions, run on roughly consecutive weekdays; four sessions of aeb/aed and four of bae/dae. In each session, a different adapting stimulus, /bae/, $/ \mathrm{dae} /, / \mathrm{aeb} /$, or $/ \mathrm{aed} /$, was used. The sessions were divided into two parts: measurement of the unadapted boundary and measurement of the adapted boundary. The unadapted boundary was found by presenting each of the seven test stimuli 32 times, in semirandom order, for identification as either " $B$ " or "D." There were about $2 \frac{1 / 4}{4} \mathrm{sec}$ between stimuli for a written response and $10 \mathrm{sec}$ between blocks of 60 . The phoneme boundary was defined as the point on the 0 to 8 stimulus scale which, by extrapolation, would receive an equal number of " $B$ " and " $D$ " responses. The adapted boundary was measured in the same way, except that a minute ( 95 presentations) of adapting stimulation preceded every four test stimuli, until each test 
stimulus had been presented 21 times. An experimental session took about $1 \mathrm{~h}, 25 \mathrm{~min}$. During adaptation, Ss were permit ted to doodle but not to read.

Care was taken to stabilize the Ss before serious testing began. Each $S$ was tested twice on both series (same procedure as unadapted testing) before any experimental sessions were run. Also, at the very beginning of each session, eight presentations of each stimulus were given as a warm-up. The aeb/aed series was used first; the order in which the adapting sounds were assigned to the daily sessions was $/ \mathrm{aeb} /, / \mathrm{aed} /, / \mathrm{dae} /, / \mathrm{bae} /$, and then $/ \mathrm{dae} /, / \mathrm{bae} /, / \mathrm{aed} /, / \mathrm{aeb} /$, for the bae/dae test sessions.

Subjects. Five Ss, aged between 18 and 25, volunteered for the experiment. They were paid $\$ 2.50 / \mathrm{h}$. All except TF were unaware of the purposes of the experiment.

\section{Results}

A boundary shift is taken as the difference between the adapted boundary for a given stimulus and the mean unadapted boundary of that particular $S$. The boundary shifts for the five Ss in each of the eight sessions are given in Table 1. Every boundary shift entry is either positive or negative. A positive entry in /aeb/ or / bae/ columns, for example, means that $B$ adaptation took place, i.e., the boundary shifted toward the "B" end of the test series. A negative entry in these columns would mean that $D$ adaptation occurred, a shift toward the "D" end of the test series.

Also given in Table 1 are the standard deviations of the four measures each of the bae/dae and aeb/aed unadapted boundaries. As estimates of the variability of boundary shifts, they are probably too small, since the unadapted boundary is measured under more stable circumstances and with more responses per stimulus. However, they serve as a useful check on the reliability of the whole experiment and each $S$ individually.

The boundary shift results are quite clear. Adaptation with /bae/ or /dae/ always has a positive adapting effect on the bae/dae series; and so does adapting with /aeb/or /aed/ and testing on aeb/aed. In other words, if the adapting and test sounds are from the same series, stimuli containing a / b/ cause B adaptation, and stimuli containing $/ d$ / cause $D$ adaptation. However, when adapting sounds and test sounds are from different continua, all four adapting stimuli give a slightly D-adapting effect. A convenient way of estimating the differential effects of $/ \mathrm{b} /$ and $/ \mathrm{d} /$ sounds is to sum the mean boundary shifts due to $/ \mathrm{b} /$ and $/ \mathrm{d} /$ within each of the four conditions. These are also given in Table 1 . It is clear that, in the crossed conditions, $/ \mathrm{b} /$ and $/ \mathrm{d} / \mathrm{stimuli}$ have no differential adapting effect.

The small boundary shifts toward " $D$ " in the crossed conditions are hard to explain. It is possible that the detectors for /bae/ and /dae/ are somehow affected by the onset of the /aeb/ and /aed/ adapting syllables: and, similarly, that /aeb/ and /aed/ detectors are affected by the final part of the / bae/ and / dae/ adapting stimuli.

\section{Discussion}

On this very strong test, a phonetic theory must be rejected. As adapting stimuli, $/ b /$ and $/ d /$ in final
Table 1

Experiment I: Standard Deviations of Unadapted Boundaries and Shifts in the aeb/aed and bae/dae Boundaries Due to Adaptation With $/ \mathbf{a e b} /, / \mathbf{a e d} /, / \mathrm{bae} /$, and $/ \mathrm{dae} /$

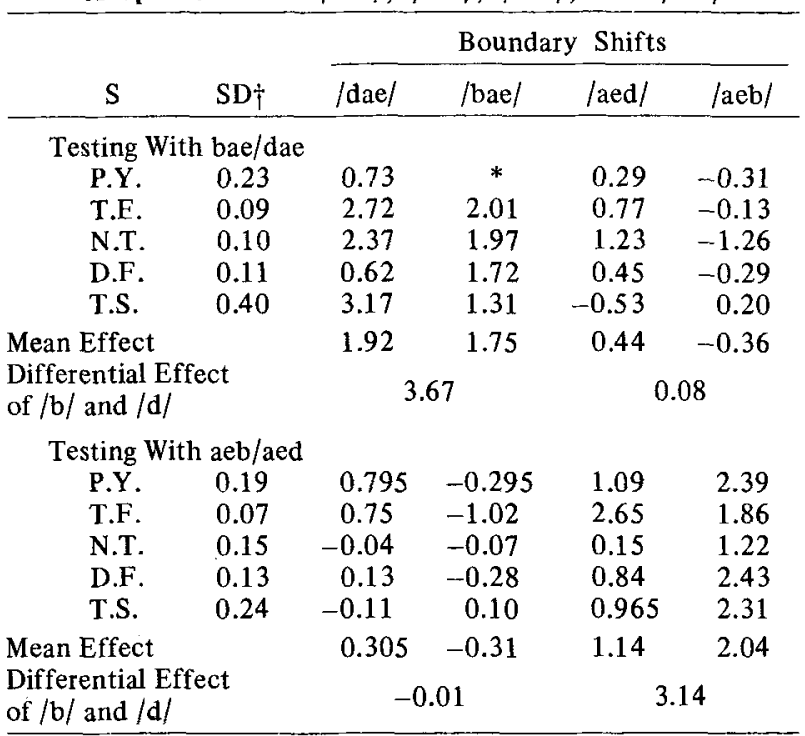

Note-All entries in stimulus units. Directions of shift are given relative to the direction predicted by the phonetic theory. " $S$ gave the same proportion of " $B$ " and " $D$ " responses to all test stimuli; his adapted boundary could not be measured. †Standard deviation of unadapted boundary $(N=4)$.

position were powerless to fatigue the detectors responsible for $/ \mathrm{b} /$ and $/ \mathrm{d} /$ in initial position, and vice versa. A truly phonetic detector would not distinguish between initial and final environments. On the other hand, the data also rule out two possible auditory theories of adaptation. One theory would attribute all adaptation effects to the desensitization of very simple mechanisms that register the energy at various frequencies. Since $/ \mathrm{aeb} /$ and $/ \mathrm{bae} /$, for example, are spectrally identical for such a mechanism, this theory actually makes the same predictions as a phonetic theory, and must be dismissed. The other auditory theory, which was mentioned above, postulates selective detectors for rising and falling temporal patterns in the second and third formants. On this theory, initial $/ \mathrm{b} /$ and final /d/ could be registered by the same "rising transition" detector. There was no trend in this direction. We can avoid the theoretical vacuum caused by the simultaneous demise of three hypotheses by concluding that there are different detectors for consonants in initial and final position. Whether or not these detectors operate in a phonetic or auditory fashion within their separate provinces is the subject of the second experiment.

\section{EXPERIMENT II /d/ WITH RISING vs FALLING TRANSITIONS}

In this experiment, a bae/dae test series was again 


Stimuli $\quad$ Initial Frequency, $\mathrm{Hz}$

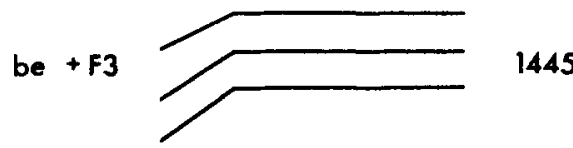

2422

be $-F 3$

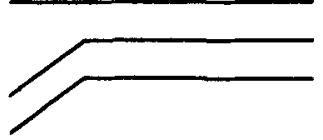

1445

2751

de $-F 3$

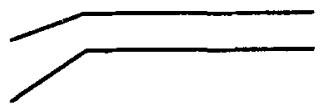

1875

2751

1959

2751

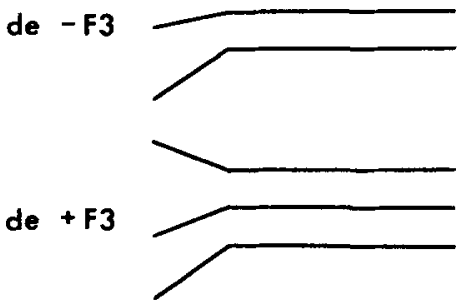

1875

3114

Fig. 2. Schematic spectrograms of the five adapting stimuli used in Experiment II. They are arranged in order of increasing D-likeness.

used. The adapting sounds were various synthesized examples of the syllables $/ \mathrm{be} /(/ \mathrm{e} /$ as in ape $)$ and $/ \mathrm{de} /$, as sketched in Fig. 2. Two of these stimuli have a formant structure typically found in natural speech: $/ \mathrm{be} /+\mathrm{F} 3$ has rising transitions in both higher formants, and $/ \mathrm{d} /+\mathrm{F} 3$ a slightly rising $F 2$ transition and a falling $F 3$. All the other sounds have a flat F3 transition (-F3). Of these, /be/ -F3 has a steeply rising $\mathrm{F} 2$ transition, /de/ $-\mathrm{F} 3$ 1875 a somewhat rising $\mathrm{F} 2$, the same as in $/ \mathrm{de} /+\mathrm{F} 3$, and /de/ -F3-1959 has a very shallow, rising F2. The logic of this design lies in the fact that the extreme/dae/ in the test series has both higher formant transitions falling. If the detectors for Cs in CV context are phoneme, or feature, specific, then all three of the adapting stimuli /de/ should be equally effective in D-adapting the bae/dae test series. If, on the other hand, the detectors are tuned to rising vs falling patterns of frequency change, then only /de/ $+F 3$ will have a D-adapting effect. The other two examples of /de/, with only rising energy patterns, will be quite ineffective as D adaptors. In fact, if there are auditory detectors for rising vs falling frequency changes, these stimuli might even be expected to B-adapt the test series. The /be/ stimuli serve to control for the possible adapting effects of the steady state vowel.
Method

Stimuli. The test stimuli used in this experiment are the same as the bae/dae series of Experiment I, except that the transitions were linear, not curved. The adapting sounds (Fig. 2) were the same as the test sounds in all respects apart from the formant frequencies. The steady state formant frequencies were 580 , 2,048 , and $2,751 \mathrm{~Hz}$.

The flat F3 transitions in two of the /de/ stimuli are not an integral part of the design. In fact, the author had intended to use two formant sounds in these cases, but was unable to synthesize a /d/ with a rising transition in F2 without F3. Attempts to do this invariably resulted in $/ \mathrm{b} / \mathrm{s}$.

Procedure and Subjects. The boundary shift procedure was exactly as described for Experiment I. There were five experimental sessions, run no closer than $24 \mathrm{~h}$ apart, one session for each adapting stimulus. Five new, naive volunteer Ss were used, aged between 18 and 22. During the adaptation period, Ss were asked to write down on a card what the adapting sounds most resembled, once at the very start of the adapting period, once at the end, and twice in the middle. Some Ss identified these sounds in rather unexpected ways. Accordingly, the five adapting sounds were presented in random order for identification. In one case, they were asked to write down "what the syllable sounds most like"; it was stressed that they need not restrict them selves to " $B$ " and " $D$ " responses. In another test, Ss were required to rate the sounds for "B-likeness" and "Dlikeness" on a 10-point scale from B-5 (most B-like) to D-5 (most D-like). These tests were administered at the start of the last four experimental sessions. In all, there were 44 presentations of each stimulus in each type of test.

\section{Results}

Table 2 gives the boundary shifts for each $S$ in each condition. Once again, the shifts are given relative to the direction predicted by the phonetic theory. Although the effects are much smaller than in Experiment $I$, the preponderance of positive entries make it clear that they exist. Indeed, there is only one case, S D.H. adapted with $/ \mathrm{be} /+\mathrm{F} 3$, where the boundary is shifted in the direction contrary to that predicted by the phonetic theory. Of immediate interest is the fact that both the /de/ $-F 3$ stimuli cause D adaptation ( $p=0.031$ in each case, by sign test). The D-adapting effect of $/ \mathrm{de} /+\mathrm{F} 3$ was, however, stronger than either the examples of $/ \mathrm{de} /$ $-F 3(p=0.031$ in each case, by sign test). There does not appear to be any difference between /be/ $+F 3$ and /be/-F3.

The results of the ranking and identification tests on the five adapting stimuli turned out to be important for the interpretation of the above results. Ss differed on how D-like or B-like they considered the adapting stimuli to be. One S, D.H., ranked all the /de/stimuli as equally D-like, but the others distinguished between them, /de/ -F3-1875 being the least D-like and/de/ +F3 the most. A similar pattern was reflected by the identification scores, except that S D.I. heard all the /de/ stimuli as "le," a fact which, surprisingly, bore no relation to his boundary shifts.

The ranking scores were compressed from 10 partitions into 5 , and the coefficients, $1,2,3,4,5$, were multiplied by the number of ranking responses in each partition. The products were then summed into a sort of quotient of D- or B-likeness. Overall, there was a very 
Table 2

Experiment 'II: Standard Deviations of Unadapted Boundaries and Shifts in the bae/dae Boundary Due to Adaptation With the /be/ and /de/ Syllables

\begin{tabular}{|c|c|c|c|c|c|c|}
\hline \multirow[b]{2}{*}{$S$} & \multirow[b]{2}{*}{$\begin{array}{c}\text { SD of } \\
\text { Unadapted } \\
\text { Boundary } \\
(\mathrm{N}=5)\end{array}$} & \multicolumn{5}{|c|}{ Adapting Stimuli } \\
\hline & & $\begin{array}{l}\text { /be/ } \\
+\mathrm{F} 3\end{array}$ & $\begin{array}{l}\text { /be/ } \\
-\mathrm{F} 3\end{array}$ & $\begin{array}{c}/ \mathrm{de} / \\
-\mathrm{F} 3 \\
\mathrm{~F} 2: 1875\end{array}$ & $\begin{array}{c}/ \mathrm{de} / \\
-\mathrm{F} 3 \\
\mathrm{~F} 2: 1959\end{array}$ & $\begin{array}{l}/ \mathrm{de} / \\
+\mathrm{F} 3\end{array}$ \\
\hline S.B. & 0.19 & 0.84 & 0.20 & 0.60 & 1.18 & 1.20 \\
\hline D.I. & 0.17 & 0.60 & 0.245 & 0.68 & 0.58 & 0.98 \\
\hline J.C. & 0.10 & 0.795 & 0.545 & 0.39 & 0.19 & 0.90 \\
\hline R.G. & 0.07 & 0.64 & 1.70 & 0.01 & 0.52 & 0.635 \\
\hline D.H. & 0.38 & -0.62 & 0.45 & 0.74 & 0.80 & 1.49 \\
\hline Mean & & 0.45 & 0.63 & 0.48 & 0.65 & 1.04 \\
\hline
\end{tabular}

Note-Direction of shift is given relative to the phonetic prediction.

high correlation between this quotient and the boundary shifts $\left(r_{s}=0.79, N=25, p<.01\right)$. In other words, the more B-like or D-like the stimulus, the greater its adapting effect.

\section{Discussion}

Since both examples of /de/ $-F 3$ had a D-adapting effect, we must conclude that there is a phonetic component to the adaptation; the /dae/ on the test continuum had a very different formant transition structure. ${ }^{1}$ An auditory detector sensitive to transitional slopes might have been effectively B-adapted by the rising F2 in these stimuli. It would be tempting to go further and claim that there must be an auditory component also because $/ \mathrm{de} /+\mathrm{F} 3$ had a greater D-adapting effect than either example of $/ \mathrm{de} /-\mathrm{F} 3$. However, since /de/ +F3 was ranked and identified as more D-like than the others, the magnitude of its adapting effect could be attributed either to its auditory representation of falling F3 frequency or to the "D-likeness" of its phonetic representation. We cannot, therefore, decide one way or the other whether there is an auditory component in addition to the phonetic one that is clearly present.

\section{CONCLUSIONS}

This paper began by noting the variability in the acoustic properties of consonants in different environments. This lack of invariance, most marked when we consider the sufficient cues to place of articulation, leads us to think of a phonetic description of speech as abstract. We have asked whether selective adaptation reveals a phonetic level which is abstract in this sense, or whether instead adaptation occurs at a lower auditory level.

The outcome of the experiments sets upper and lower bounds on the abstractness of the adapted level. It must be lower than a level where consonants are represented as the same irrespective of syllable position (Experiment I), but high enough to represent phonemes in initial position as the same irrespective of the broad acoustic differences conditioned by the following vowel.
This second assertion of an abstract phonetic description within syllable position must be regarded as only tentative at this stage. Firstly, the adapting effect of /de/ on the mechanisms responsible for /dae/ was quite small, only $30 \%$ of the adapting effect of /dae/ itself. If a phonetic level was all that was involved, we would expect complete transfer, in spite of acoustic differences. Secondly, I have taken /de/ and /dae/ as acoustically different because their transitions are of opposite slope. If, instead, the absolute frequency of the transitions, or their starting frequency, or the frequency locus to which they point, are considered to be the critical cues, then /de/ is acoustically similar to /dae/. It must be admitted, therefore, that each of these three possibilities constitutes an alternative explanation of Experiment II in terms of auditory rather than phonetic similarity. While none of these alternativs can explain Experiment I unless we build into them a dependency on syllable position, they clearly deserve further attention.

In summary, then, adaptation cannot be phonetic in a truly linguistic sense: phonemes and distinctive features are the same in linguistic theory in any position, while adaptation is entirely position dependent. On the other hand, within a given position, detectors for quite abstract properties, probably phonetic, can be revealed by selective adaptation.

\section{APPENDIX}

The stimuli were all synthesized on the Speech Communications Group PDP-9 computer facility at the Research Laboratory of Electronics, M.I.T., using a program written by D. H. Klatt. The program simulated a parallel resonance synthesizer. The program also arranged for the output of the stimuli in a format appropriate to identification testing and the making of tape loops.

The steady state vowel formant frequencies, in the order $\mathrm{F} 1,2,3$, were $742,1,618$, and $2,751 \mathrm{~Hz}$; formant amplitudes relative to $\mathrm{F} 1$ were $-6 \mathrm{~dB}$ for $\mathrm{F} 2$ and $-17 \mathrm{~dB}$ for $\mathrm{F} 3$; formant bandwidths were 50,79 , $118 \mathrm{~Hz} /$ half power. The steady state was $230 \mathrm{msec}$ in duration, and the transitions, $35 \mathrm{msec}$. The fundamental 
frequency, the same in both initial and final series, rose from 95 to $104 \mathrm{~Hz}$ in the first $60 \mathrm{msec}$, and fell linearly to $85 \mathrm{~Hz}$. The overall amplitude also fell off slowly in the last $30 \mathrm{msec}$ of the $\mathrm{CV}$ series, to make the stimuli sound more natural. The onset of the $\mathrm{VC}$ syllable was also slightly contoured.

The extreme frequencies of $\mathrm{F} 2$ and F3 transitions were 1,384 and $2,422 \mathrm{~Hz}$ in the extreme $/ \mathrm{b} /$, and 1,696 and $3,114 \mathrm{~Hz}$, respectively, in the extreme $/ \mathrm{d} / \mathrm{s}$. The amplitude of the formants during transition followed the acoustical theory of Fant (1956). Between these extreme values, the stimuli were spaced apart in the ratio of $2,1,1,1,1,2$, differing only in transition direction.

\section{REFERENCES}

Cole, R. A., \& Scott, B. Acoustic invariance for stop consonants. Paper presented at the 82 nd meeting of the Acoustical Society of America, Denver, 1971.

Corst, J, M, \& Gerstman, L. J. Some experiments on the perception of synthetic speech sounds. Journal of the Acoustical Society of America, 1952, 24, 597-606.

Cooper, W. E. Adaptation of phonetic feature analysers for place of articulation. Journal of the Acoustical Society of America, in press.

Cooper, W. E., \& Blumstein, S. E. A "labial" feature analyzer in speech perception. Perception \& Psychophysics, 1974, 15, 591-600.

Delattre, P. C., Liberman, A. M., \& Cooper, F. S. Acoustic loci and transitional cues for consonants. Journal of the Acoustical Society of America, 1955, 27, 769-773.

Eimas, P. D., \& Corbit, J. D. Selective adaptation of linguistic feature detectors. Cognitive Psy chology, 1973, 4, 99-109.

Fant, C. G. M. On the predictability of formant levels and spectrum envelopes from formant frequencies. In I. Lehiste (Ed,), Reading in acoust ic phonetics. Cambridge, Mass: M.I.T. Press, 1967.

Fischer-Jorgensen, E. Acoustic analysis of stop consonants. Miscellanea Phonetica, 1954, 2, 43-59.

Fischer-Jorgensen, E. Perceptual studies of Danish stop consonants. Annual Report No.6 of the Institute of Phonetics, University of Copenhagen, 1972, 75-176.

Halle, M., Hughes, G. W., \& Radley, J.-P. A. Acoustic properties of stop consonants. Journal of the Acoustical Society of America, 1957, 27, 107-179.

Lisker, L., \& Abramson, A. S. The voicing dimension: Some experiments in comparative phonetics. Proceedings of the 6th International Congress of Phonetic Sciences, Prague, 1967. Academic Publishing House of the Czechoslovak Academy of Sciences, 1970 .

Stevens, $K$. N. The potential role of property detectors in the perception of consonants. Paper presented at Symposium on Auditory A naly sis \& Perception of Speech, Leningrad, August 1973.

Stevens, K. N., \& Klatt, D. H. The role of formant transitions in the voice-voiceless distinction for stops. Quarterly Progress Report No. 101, Research Laboratory of Electronics, M.I.T., 1971.

\section{NOTE}

1. Professor K. N. Stevens raised the possibility that the onsets of $\mathrm{F} 2$ and $\mathrm{F} 3$ might have been sufficiently sudden to cause high-frequency energy at the beginning of the two $/ \mathrm{de} /-\mathrm{F3}$ syllables. If this was the case, they would have had a simple acoustic attribute in common with the /dae/ of the test series. initial high frequency. Professor $W$. L. Henke was kind enough to prepare Fourier analyses of the first $10 \mathrm{msec}$ of each of the five adapting stimuli. There did not appear to be unwanted high-frequency components that would account for the results.

(R eceived for publication November 16, 1973; revision received February 12,1974 